Biorheology 30, V, 1993

$0006-355 \mathrm{X} / 93 \$ 6.00+.00$ Printed in the USA.

Copyright $\odot 1993$ Pergamon Press Ltd. All rights reserved.

BIORHEOLOGY

\title{
AUTHOR INDEX, VOLUME 30, 1993
}

Agarwal, M., 49

Baaijens, J. P. W., 63

Barthes-Biesel, D., 1

Beritashvili, N., 153

Brookshier, K. A., 107

Bucherer, C., 1

Casley-Smith, J. R., 9, 93

Chandran, K. B., 117

Charonsonney, O., 75

Cloutier, G., 443

De Smedt, S. C., 31

Dekeyser, P., 31

Dellsperger, K., 371

Demeester, J., 31

Djabourov, M., 191

Donath, E., 463

Drochon, A., 1

Dufaux, J., 75

Duvelleroy, M., 75

Edwards, D. H., 333

Elkin, G., 43

Gaill, F., 191

Goldsmith, H. L., 165

Gomez, J. E., 409

Gosline, J. M., 229

Goto, M., 323

Gratzer, W. B., 397

Griffith, T. M., 333

Hasegawa, S., 275

Hofmann, K. E., 243

Hori, M., 349, 359

Inoue, M., 359

Janssen, J. D., 63

Kajiya, F., 323

Kamada, T., 359

Kanatsuka, H., 371

Kawai, M., 435
Kim, Y. H., 117

Kimura, A.., 323

King, M. 49

Kitakaze, M., 359

Kohyama, K., 243

Komaru, T., 371

Lacombe, C., 1

Lauwers, A., 31

Lechaire, J.-P., 191

Lelièvre, J. C., 1

Lillie, M. A., 229

Lim, Y.-J., 349

Lominadze, D., 153

Löw, M., 287

Masuda, T., 435

Masuyama, T., 349

Matsumoto, T., 435

Matsuo, T., 267

Mchedlishvili, G., 153

Minamino, T., 359

Morioka, T., 359

Moritaka, H., 243

Mouren, S., 75

Nakamura, K, 207

Nanto, S., 349

Nash, G. B., 397

Nieuw Amerongen, A. V., 141

Niki, R., 207

Nishinari, K, 243

Nishinari, N., 243

Oddou, C., 387

Ohshima, N., 131

Okazaki, Y., 359

Okeda, R., 267

Okino, H., 429

Ookawa, K., 131
Pastushenko, V. F., 463

Perktold, K, 287

Pomorski, T., 463

Quemada, D., 253

Raunig, R., 287

Razakamiadana, A., 387

Ribitsch, V., 31

Rodgers, G. P., 275

Ross-Murphy, S. B., 217

Sato, H., 359

Sato, M., 131

Schechter, A. N., 275

Shatwell, K. P., 217

Shio, H., 275

Shukla, J. B., 49

Shung, K. K., 443

Sipkema, P., 381

Stoltz, J. F., 305

Takashima, S., 359

Takashima, T., 371

Tarbell, J. M., 107

Taylor, T., 429

Thurston,, G. B., 409

Tsinamdzrishvili, B., 153

Tsujioka, K., 323

Uyesaka, N., 275

van der Linden, P. J. W., 381

van der Reijden, W. A., 141

van Steenhoven, A. A., 63

Veerman, C. C. I., 141

Vicant, E., 75

Watase, M., 243

Weiss, L., 43

Westerhof, N., 381

Yamaguchi, T., 429

Yamamoto, K., 267

Zahalak, G. I., 471 